\title{
Document Logical Structure Analysis Based on Perceptive Cycles
}

\author{
Yves Rangoni and Abdel Belaïd \\ Loria Research Center - Read Group, Vandœuvre-lès-Nancy, France \\ \{rangoni, abelaid\}@loria.fr \\ http://www.loria.fr/ rangoni/ \\ http://www.loria.fr/ abelaid/
}

\begin{abstract}
This paper describes a Neural Network (NN) approach for logical document structure extraction. In this NN architecture, called Transparent Neural Network (TNN), the document structure is stretched along the layers, allowing an interpretation decomposition from physical (NN input) to logical (NN output) level. The intermediate layers represent successive interpretation steps. Each neuron is apparent and associated to a logical element. The recognition proceeds by repetitive perceptive cycles propagating the information through the layers. In case of low recognition rate, an enhancement is achieved by error backpropagation leading to correct or pick up a more adapted input feature subset. Several feature subsets are created using a modified filter method. The first experiments performed on scientific documents are encouraging.
\end{abstract}

\section{Introduction}

This paper tackles the problem of document logical structure extraction based on physical feature observations within document images. Although this problem has known many solutions, it still remains very challenging for noisy and variable documents.

The literature abounds of structure analysis approaches for different document classes. A survey of the most important approaches can be found in [1]. Most of them are based on formal grammars describing the connection between logical elements. However, these methods have drawbacks because the rules are given by the user and could be not sufficient to handle complex and noisy documents. It is difficult to remove ambiguities and many thresholds must be fixed to process the matching between the physical and the logical structure.

Consequently, a learning-based method seems to be a more adapted solution. Artificial neural network (ANN) approaches allow such a training (rules are learnt) and are known to be more robust to noise and deformation. However, ANN like the classical Multi Layer Perceptron (MLP) is considered as a black box and does not explicit the relationships between the neurons. In the same time, domain-specific knowledge appears essential for document interpretation as mentioned in [2] and it seems useful to keep a part of knowledge in a Document Image Analysis (DIA) system. 
In order to take into account theses two aspects (knowledge and learning), we propose a new ANN approach that use a Transparent Neural Network (TNN) architecture. This method has the same MLP capacities and can act, in the same time, on the reasoning by introducing knowledge. The recognition task is done progressively by propagation of the inputs (local vision) towards the outputs (global vision). Back-propagation movements, during recognition step, are used for an input correction process as the human perception acts. These successive "perceptive cycles" (vision-interpretation) bring a context return which is very helpful for the input improvement.

This paper is organized as follows. In the first section, the TNN architecture is described. The second section details an input feature clustering method to speed up the perceptive cycles. Finally, in the last section experimental results and discussions are reported.

\section{The TNN Architecture Description}

The proposed TNN architecture is described in Fig. 1. The first layer receives physical features where each element corresponds to a neuron. The following layers represent the logical structure at three different levels, from fine to coarse (see Fig 8 for the whole input and output names).

All the layers are fully connected and all the neurons carry interpretable concepts. This modeling integrates common knowledge on "general" document

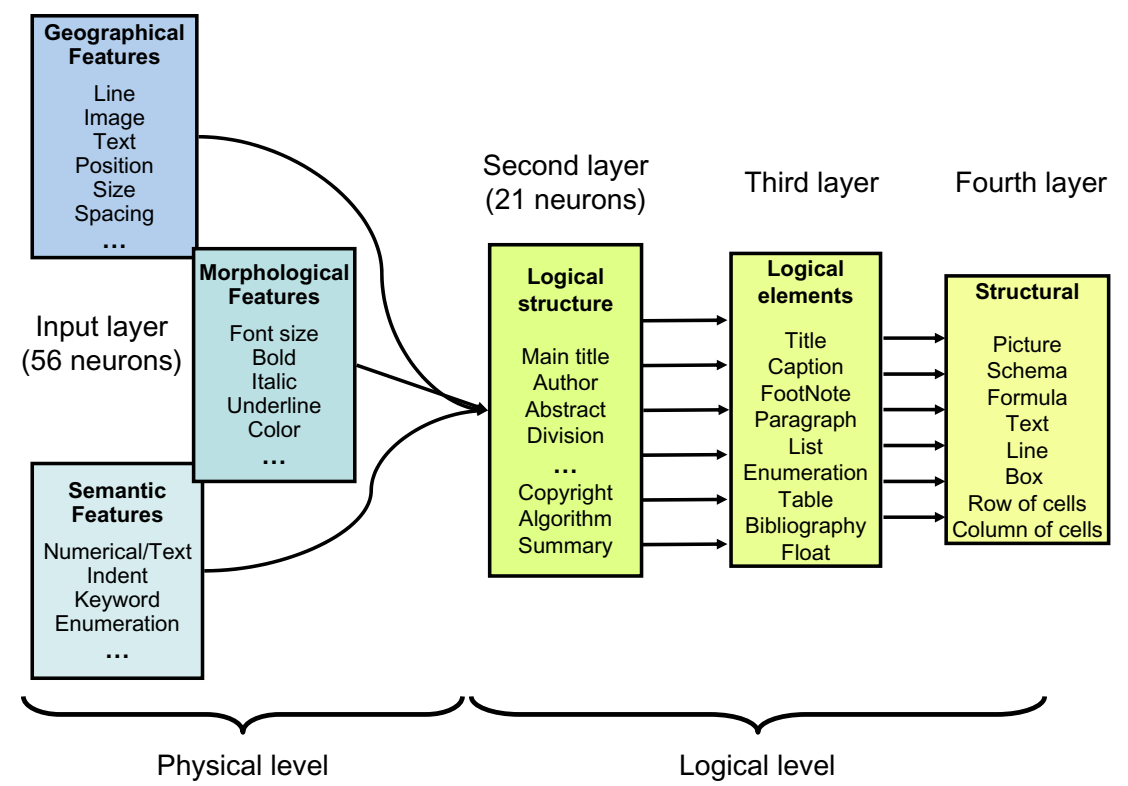

Fig. 1. Neuron semantic for document analysis 
structure. It can be more precise if a DTD (Document Type Definition) is given as the DTD organizes the logical element in hierarchy. The real TNN output is the first logical level (the second layer) while the last layers represent the global context (third and fourth layers). They are used to precise the context which is needed for logical structure identification during the perceptive cycles.

This system can be considered as a hybrid method set between a modeldriven (DTD integration) and a data-driven approach (training phase). As for a classical MLP, a database is used to train the links between physical and final logical structures. The list of input and output elements is given in Fig. 8 ,

As the model is transparent and errors can be known for each output layers, the training of the whole network is achieved locally for each consecutive layer pairs. The weight modifications are carried out by an error correction principle. The first stage consists in initializing the weights with random values, then for each couple (Input, Output) of the training database, a predicted value is computed by propagation. The error between the computed output $P$ and the desired output $O$ is then determined. The second stage back-propagates this error in the previous layers. If the activation function is a sigmoid, the value to add to a weight $w_{i, j}$ is $\alpha\left(O_{j}-P_{i}\right) P_{i}\left(1-P_{j}\right) I_{i}$.

The Fig. 2 shows a small example of a trained TNN that classifies RGB colors in rainbow colors. The green links are used for a positive contribution and the red ones are used for a negative one. The line thickness is proportional to the link weight.

Contrary to a MLP, the recognition process is more complicated. The MLP looks at the maximum output layer component $O_{i}=\operatorname{argmax}\{O\}$ and deduces that the input pattern belongs the $i^{\text {th }}$ class. In a TNN system, the outputs are analyzed and two decisions can be chosen (Fig. 3.):

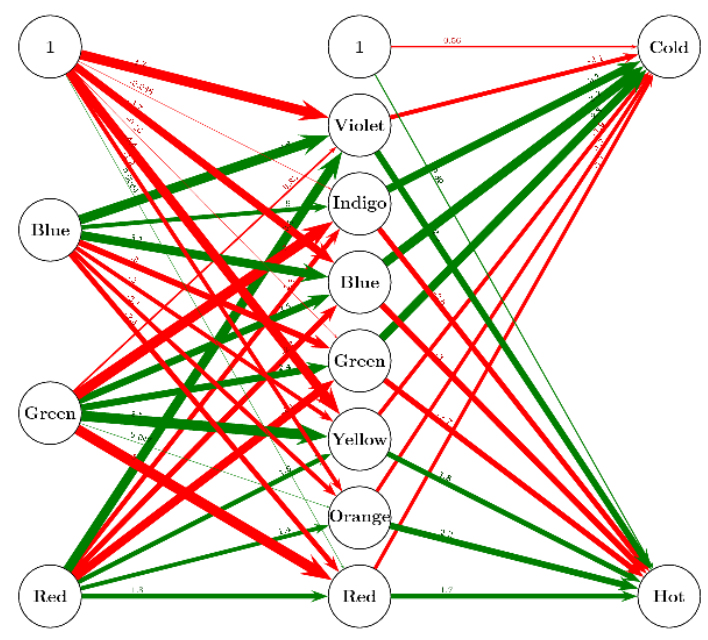

Fig. 2. A TNN classifying RGB pixels in hot and cold colors. It uses rainbow color decomposition in its intermediary layer. 


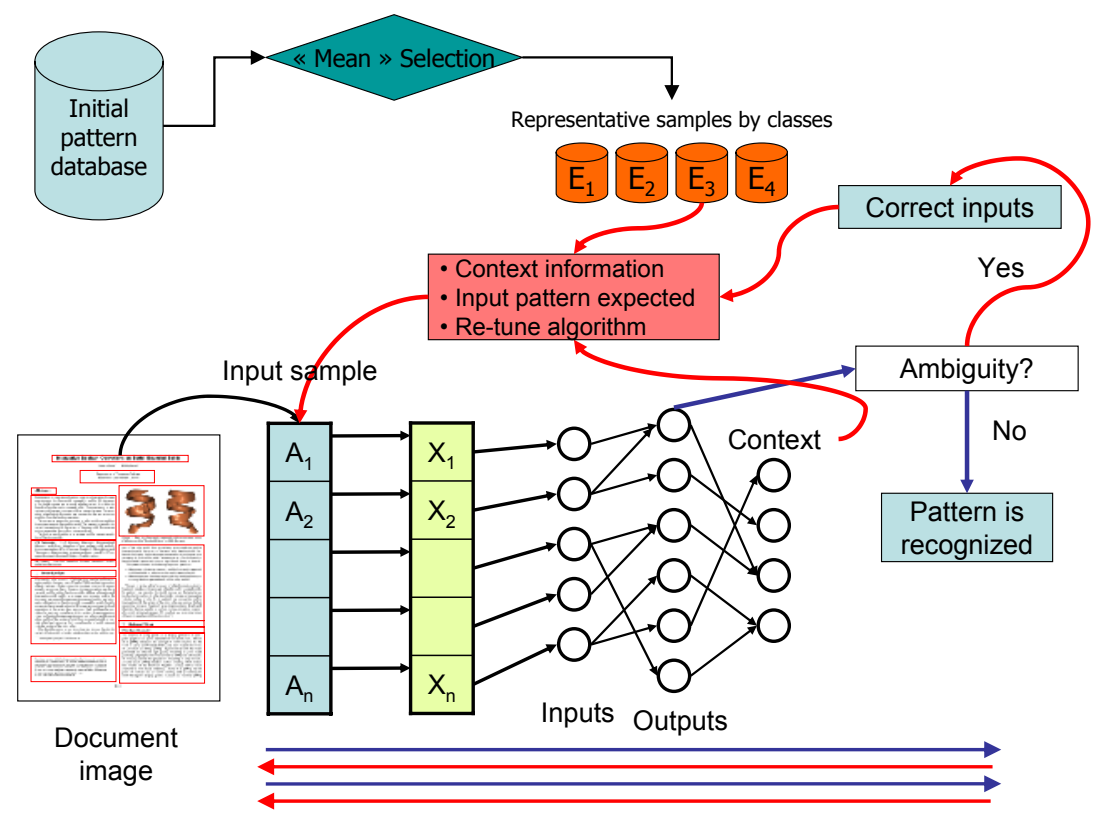

Fig. 3. Perceptive cycles: propagation, analysis, context return, and correction

- the first decision concerns the output when it is close to a unit vector. Thus, the system gives a ruling on a "good" pattern. This means that a class has a sufficient score $\|O\|_{\infty} \geqslant \varepsilon$ with $0 \ll \varepsilon<1$ (acceptable class) and this winning class has a score greater than the others $\Gamma(O)=\frac{n\left(\left(\sum O_{i}\right)^{2}-\sum O_{i}^{2}\right)}{(n-1)\left(\sum O_{i}\right)^{2}} \leqslant \eta$ with $0<\eta \ll 1$ (superior class). If such an output satisfies these rules, the system stops and the pattern is classified.

- the alternative decision occurs when the system reports an ambiguity (i.e. the pattern is confused among several classes). In that case, the latest TNN layers react and propose a context. Thanks to the known neuron semantic, information from upper layers are used to determine the possible or unlikely classes. A hypothesis is created about the possible pattern class and then the input is analyzed in order to find the wrong component values.

As the input physical features (e.g. bounding box, font style, text, etc.) are determined by specific algorithms, it is possible to operate on their precision (or quality) by reconsidering the algorithm parameters, or by changing totally the algorithm method. An example of "re-tuning" can be the OCR settings that give the text. It is possible in an OCR engine to change the amount of computation but changing consequently the recognition quality. The "High Speed" mode is chosen when it is needed to separate text and image whereas "High Quality" mode is preferred if a precise word (a key-word for example) is searched in the text block.

Another example of algorithm "swapping" is the evaluation of word number in a text block. Two solutions can be chosen. The first solution uses RLSA and 
(a)

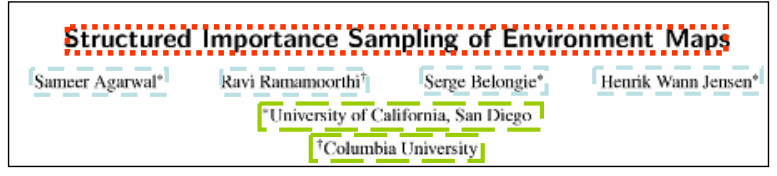

(b)

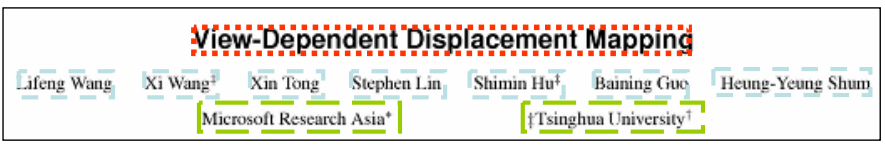

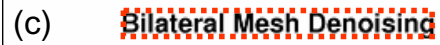

Shachar Teishran - Iddo Drới - Daniel Cohen-Or School of Computer Science, Tel Aviv University*

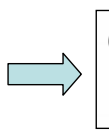

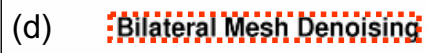

[Shachar Fleishman]
[School of Compuler Science, Tel Aviv University"]

Fig. 4. In (a) and (b), the segmentation and labeling results are correct. Picture (c) shows the result before a context return: the authors are not found. In (d) is the recognition process of (c) after an input correction: title, authors, and locality are found now.

evaluates the number of connected components. The second solution uses an OCR and simply counts the number of words. The first solution is the fastest but gives approximate results whereas the second solution is more time-consuming but more accurate.

With the use of context, new information coming from the training database can be added during the correction. For example, if a segmentation problem occurs, the system finds the "mean" awaited bounding box and corrects the previous bounding box dimensions. This example is not insignificant, because segmentation errors are frequent and penalize the whole physical extraction. The context returns allow often a better segmentation and contribute to better recognition accuracy (see Fig. 4).

\section{Input Feature Clustering}

In the previous section, the TNN is showed to be able to analyze its outputs and to improve its inputs accordingly. Better results than a MLP can be obtained thanks to the perceptive cycles. However, "high-level" feature extraction is needed for each cycle what remains a time-consuming procedure.

In order to speed-up the global process, the input features are categorized and classified in subsets. The feature subsets are used progressively as TNN inputs. A first feature set is chosen and then if the recognition rate is too low, another set (containing the first and additional features) is selected and so on until reaching the final solution. As the whole features are not necessary needed to classify many patterns the computation is reduced consequently.

Two criteria are used for feature classification: "quality" and "velocity". The "velocity" corresponds to the algorithm execution time given either by experiments or formally by studying the algorithm complexity. For "quality", there is no straightforward measurement method. A specific method based on feature 


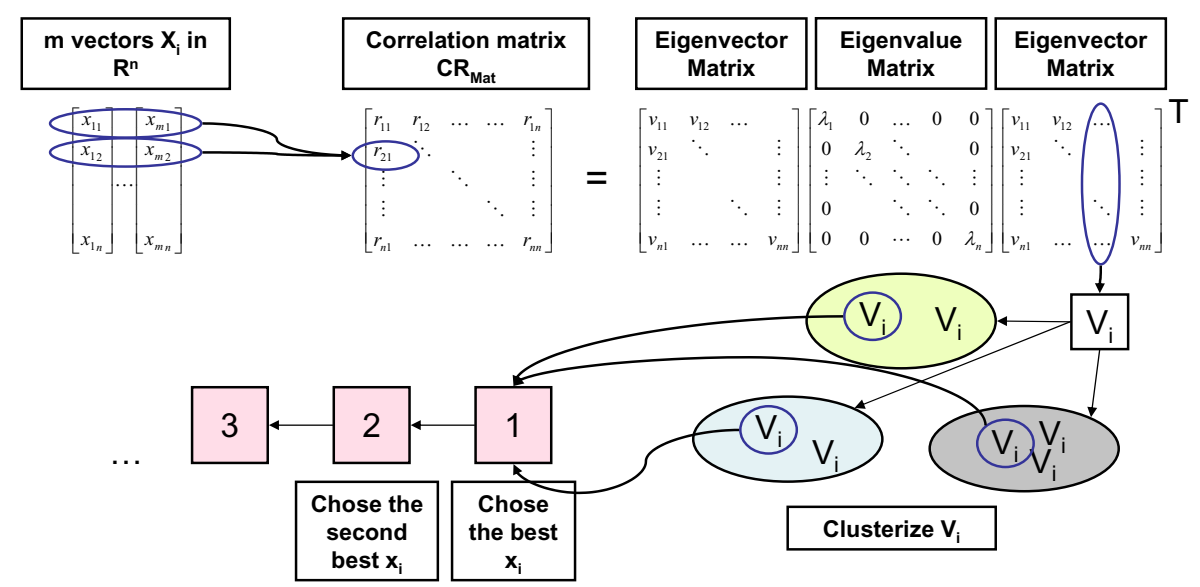

Fig. 5. Data categorization according to predictive capacity

subset selection is proposed. The objective is to determine the best feature combination to feed a pattern classification system. This method is used to create a feature partitioning.

The literature mentions two main feature selection methods: filter and wrapper 3. The first one selects variables by ranking them with correlation coefficients (it is usually suboptimal for building a predictor, particularly if the variables are redundant). The second one assesses variable subsets according to their usefulness to a given predictor (but the predictor is needed to construct the subsets). As the subsets are needed to construct the TNN architecture, a filter method has been considered. The filter method is also adapted to exclude many redundant variables in the same subset and to keep the most relevant ones.

The Karhunen-Loeve transform is used as a first step in the filter selection method. In 4 we used an extension of the Principal Component Analysis (PCA) in order to build subsets of initial features and not rewrite the features in another base, as the PCA is originally designed.

The eigenvectors $V$ (in absolute value) of the data correlation matrix $C R_{M a t}=$ $\left(\operatorname{cor}\left(X_{i}, X_{j}\right)\right)$ are computed. The vectors are then clusterized using a Self Organizing Map (SOM) with an Euclidian distance (see Fig. 5.).

The obtained clusters contain similar eigenvectors (i.e. redundant variables). The feature corresponding to the nearest eigenvector from each cluster center is chosen for the creation of a new subset. This subset contains high predictive features which are the least correlated. By fixing the neuron number of the SOM, the number of desired subsets can be chosen.

An important phase of this clustering process is to determine the lower-space dimension $q$ (i.e. the variance to be kept). As no optimal solution exists, some heuristics proposed by the literature have been tested: 
- fixed number $q$ : this is a straightforward method where cutting level is imposed by the user.

- fixed percentage: similarly to the previous case, but here the user chooses the first $p \%$ of the eigenvalues.

- cumulated percentage: the number $q$ is determined when the sum of the first variance (eigenvalue) is greater than a given fixed percentage.

These three methods are usual but their choice assumes that the user overcomes its application and can appreciate the dimension to use. These methods are often used in social sciences because it is easier to interpret the data. Two other methods, which are more general and more robust, are based on the shape of the eigenvalue sequence:

- Kaiser method: the average of all the variances is calculated. The space dimension $q$ is determined when the sum of the first variance is greater than this average. Of a wide spread employment, it can be put at fault.

- Cattell method: [5] suggests to find the place where the smooth decrease of eigenvalues appears to level off to the right of the plot (the scree-test). This heuristic is often considered as the most powerful [6]

\section{Experimental Results and Discussion}

Before introducing results on document image analysis, experiments about "lowlevel" and highly correlated features are presented.

A first experimentation procedure is employed to illustrate the variable subset creation method. For this purpose, the MNIST database [7] is used. A MultiLayer Perceptron is used to evaluate the group validity. This classifier has the same settings along all the experiments (topology, initial random weights, etc.). Two experiments have been made on this database. The first uses the whole initial pixels of each image (digits are $28 \times 28$ pixel images). The second experiment uses resampled images (in a $7 \times 7$ format). Thus, we have for the first experiment 784 variables are considered in the first experiment and 49 for the second one.

The MLP is trained with different variable subsets and the recognition rate is chosen as a quality measurement. The subsets are compared to randomly created ones. One thousand of random subsets have been generated and evaluated by the MLP. Then the best one is retained for the comparison. This procedure will be the same for the following experiments about document analysis.

Table 1 shows normalized comparison results between the subset obtained by our method and the best of the random subsets for the initial MNIST database. The Fig. 6] shows the position of the selected pixels in the image. The first picture represents the "mean" digit coming from the whole database $\left(\frac{1}{n} \sum_{i=1}^{n} I_{i}\right)$ and in the next three pictures, chosen pixels can be seen. The Table 2 is similar to the Table 1 but here $7 \times 7$ pixel images are used for test.

The approach gives good results in spite of the strong influence of each pixel (expecting those on the border) on the classifier. The method keeps the two thirds of the information by keeping less than $4 \%$ of features (Table 1; with 25 of the 784 variables, $67.6 \%$ of the information is kept). 
Table 1. MNIST digit classification accuracy while decreasing the number of features

\begin{tabular}{|c||c|c|}
\hline \multicolumn{1}{|c||}{} & \multicolumn{2}{c|}{ Method } \\
\# features & Random & Our selection \\
\hline \hline $784(\max )$ & $100 \%$ & $100 \%$ \\
\hline 500 & $98.4 \%$ & $99.2 \%$ \\
\hline 300 & $95.9 \%$ & $98.4 \%$ \\
\hline 150 & $90.5 \%$ & $96.5 \%$ \\
\hline 100 & $84.2 \%$ & $94.2 \%$ \\
\hline 50 & $70.9 \%$ & $87.8 \%$ \\
\hline 25 & $47.1 \%$ & $67.6 \%$ \\
\hline
\end{tabular}

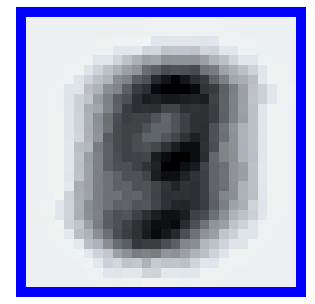

Mean digit image

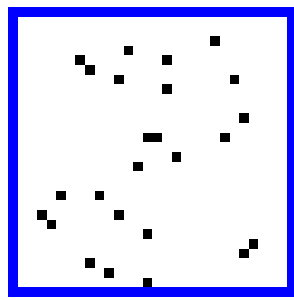

25 chosen pixels

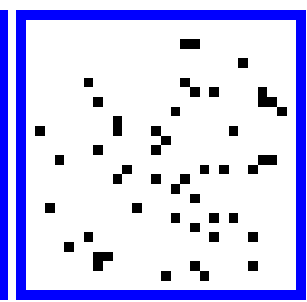

50 chosen pixels

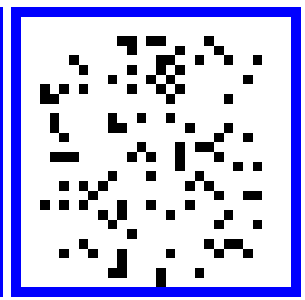

100 chosen pixels

Fig. 6. Feature subsets created for MNIST database

Experiments concerning the document logical structure analysis are presented below. We have chosen as a main database 74 Siggraph 2003 conference papers 8]. The documents are scientific articles having many and diversified logical structure elements (see Fig. 7 for two examples).

In these 74 documents, 21 logical structures are labeled that represents more than 2000 patterns. The input and output features are presented in Fig. 8 Note that all the physical inputs (geometrical, morphological, and semantic) are numerical values between 0 and 1 after possibly normalization. In general, the number represents a percentage (e.g. the percentage of bold characters in a text block) and for other features that represent a number (e.g. the number $k$ of keywords in a text block) we use the series $\sum_{n=1}^{k} 1 /(n+1)$ to have a number between 0 and 1 .

As previously, the same protocol for input feature selection is experimented on this document database. We extracted physical information from the document layout. There are 56 features composed of geometrical, typographical, and morphological information (see Fig. 8) and we use once again a MLP as classifier.

Table 3 synthesizes some results of logical structures recognition accuracy according to the eigenvalue choice methods as mentioned at the end of the previous section. The five methods have been tested on different subset sizes.

The choice of the space dimension influences the results quality. Even if the MLP is a classifier able to give good results with few features, choosing too low 
Table 2. Resampled MNIST digit classification accuracy while decreasing the number of features

\begin{tabular}{|c||c|c|}
\hline \multicolumn{1}{|c||}{} & \multicolumn{2}{c|}{ Method } \\
\# features & Random & Our selection \\
\hline \hline $49(\max )$ & $100 \%$ & $100 \%$ \\
\hline 35 & $94.2 \%$ & $99.3 \%$ \\
\hline 25 & $81.2 \%$ & $88.6 \%$ \\
\hline 15 & $56.2 \%$ & $70.5 \%$ \\
\hline 10 & $43.9 \%$ & $55.2 \%$ \\
\hline
\end{tabular}

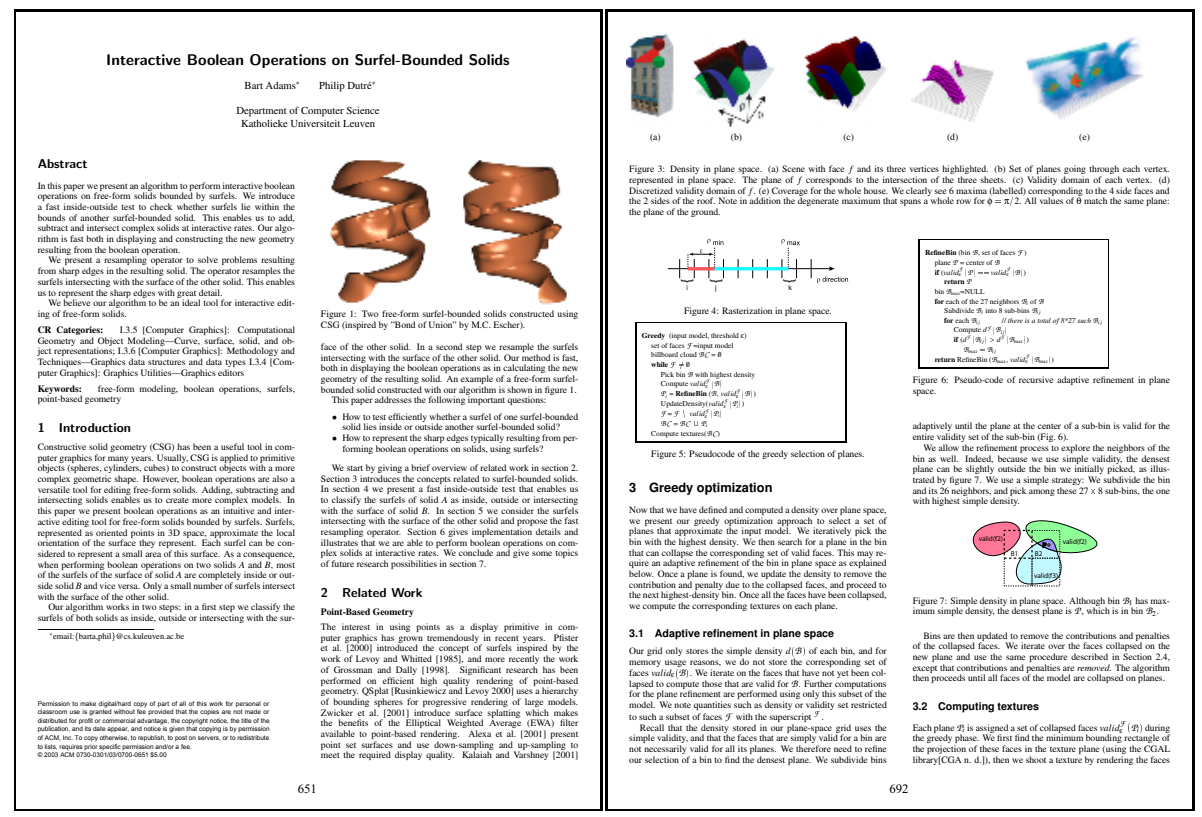

Fig. 7. Two scientific document database samples

or too high eigenvector dimension can be bad for the input feature clustering and consequently for the classifier.

It seems here (and for other tests that have be done on MNIST) that the Cattell method (that set $q=19$ ) is most of the time better than Kaiser (with $q=14$ ). The two methods, which automatically find the number $q$, give the same or better results than the classical ones where the user must fix this number. We will retain for the following tests the Cattell method that seems to be the most robust on many experimentations.

As expected and confirmed in Table 4, these "high-level" features lends well to this selection.

In this case, the choice of a small set of features is more difficult. The feature clustering method seems to be appropriate when the number of features is rather 


\begin{tabular}{|c|c|c|c|}
\hline Logical & Geometrical & Morphological & Semantic \\
\hline \hline Title & Text & Bold & IsNumeric \\
Author & Image & Italic & KeyWords \\
Email & Table & Underlined & \% KnownWords \\
Locality & Other & Strikethrough & \% Punctuation \\
Abstract & x position & UpperCase & Bullet \\
Key words & y position & Small Capitals & Enum \\
CR Categories & Width & Subscript & Language \\
Introduction & Height & Superscript & Baseline \\
Paragraph & NumPage & Font Name & \\
Section & UpSpace & Font Size & \\
SubSection & BottomSpace & Scaling & \\
SubSubSection & LeftSpace & Spacing & \\
List & RightSpace & Alignment & \\
Enumeration & & LeftIndent & \\
Float & & RightIndent & \\
Conclusion & & FirstIndent & \\
Bibliography & & NumLines & \\
Algorithms & & Boxed & \\
Copyright & & Red/Green/Blue & \\
Acknowledgments & & & \\
Page number & & & \\
\hline
\end{tabular}

Fig. 8. Logical outputs and physical inputs for documents

Table 3. Logical structure rate accuracy (in \%) according to dimensionality $q$ reducing method

\begin{tabular}{|c|c|c|c|c|c|c|c|c|}
\hline \multirow{2}{*}{$\begin{array}{l}\text { Feature } \\
\text { number }\end{array}$} & \multicolumn{2}{|c|}{ Fixed number } & \multicolumn{2}{|c|}{ Fixed \% } & \multicolumn{2}{|c|}{$\%$ Variance } & \multirow{2}{*}{$\begin{array}{l}\text { Kaiser } \\
(q=14)\end{array}$} & \multirow{2}{*}{$\begin{array}{l}\text { Cattell } \\
(q=19)\end{array}$} \\
\hline & Num & Accur. & $\mathrm{F} \%$ & Accur. & $\% \mathrm{~V}$ & Accur. & & \\
\hline \multirow{4}{*}{5} & 2 & 64.4 & $2 \%$ & 61.6 & $10 \%$ & 66.5 & \multirow{4}{*}{69.2} & \multirow{4}{*}{68.1} \\
\hline & 5 & 64.3 & $5 \%$ & 72.1 & $20 \%$ & 67.9 & & \\
\hline & 10 & 60.3 & $10 \%$ & 64.3 & $40 \%$ & 61.4 & & \\
\hline & 15 & 59.2 & $20 \%$ & 57.8 & $60 \%$ & 63.6 & & \\
\hline \multirow{4}{*}{10} & 2 & 78.4 & & 79.7 & & 81.1 & \multirow{4}{*}{77.7} & \multirow{4}{*}{82.3} \\
\hline & 5 & 79.8 & & 82.7 & & 73.5 & & \\
\hline & 10 & 72.9 & & 77.1 & & 78.4 & & \\
\hline & 15 & 70.0 & & 76.6 & & 72.6 & & \\
\hline \multirow{4}{*}{20} & 2 & 85.4 & & 82.1 & & 82.3 & \multirow{4}{*}{85.7} & \multirow{4}{*}{86.1} \\
\hline & 5 & 84.9 & & 82.8 & & 86.1 & & \\
\hline & 10 & 83.6 & & 83.3 & & 82.3 & & \\
\hline & 15 & 82.6 & & 83.3 & & 78.8 & & \\
\hline \multirow{4}{*}{30} & 2 & 85.2 & & 82.9 & & 84.2 & \multirow{4}{*}{87.4} & \multirow{4}{*}{88.0} \\
\hline & 5 & 86.8 & & 85.6 & & 85.8 & & \\
\hline & 10 & 86.6 & & 86.5 & & 86.7 & & \\
\hline & 15 & 86.3 & & 85.4 & & 87.7 & & \\
\hline
\end{tabular}

small and can be very powerful in this case (more than $83 \%$ of information is kept by dividing the variable number by 5$)$.

Leaving side input features selection, results about complete DIA system are presented. Three input features subsets are created with the previous method. 
Table 4. Logical elements classification accuracy while decreasing the number of features

\begin{tabular}{|c||c|c|}
\hline \multicolumn{1}{|c||}{} & \multicolumn{2}{c|}{ Method } \\
\# features & Random & Our selection \\
\hline \hline $56(\max )$ & $100 \%$ & $100 \%$ \\
\hline 35 & $86.9 \%$ & $99.3 \%$ \\
\hline 25 & $65.0 \%$ & $79.6 \%$ \\
\hline 15 & $51.8 \%$ & $80.1 \%$ \\
\hline 10 & $35.1 \%$ & $83.8 \%$ \\
\hline 5 & $17.9 \%$ & $44.9 \%$ \\
\hline
\end{tabular}

Table 5. Logical classification by MLP and TNN with perceptive cycles

\begin{tabular}{|c||c|c|c|c|c|}
\hline \multicolumn{1}{|c||}{ Recognition } & \multicolumn{5}{|c|}{ TNN } \\
rates & MLP & $C_{1}$ & $C_{2}$ & $C_{3}$ & $C_{4}$ \\
\hline \hline All elements & $81.6 \%$ & 45.2 & 78.9 & 90.2 & $91.7 \%$ \\
\hline Best class & $86.9 \%$ & 66.7 & 85.3 & 85.3 & $99.3 \%$ \\
\hline Worst class & $0.0 \%$ & 0.0 & 0.0 & 4.0 & $28.6 \%$ \\
\hline \hline $\begin{array}{c}\text { Recognition time } \\
\text { (MLP as reference) }\end{array}$ & $100 \%$ & $70 \%$ & $145 \%$ & $185 \%$ & $240 \%$ \\
\hline
\end{tabular}

Extraction tools, which can be configured, are used to extract the physical layout. During the recognition phase, the system can choose between the feature subsets and act on extraction tools as mentioned in Section 3. The training stage uses 44 documents and 30 for the test. Test results between a MLP and the TNN at the end of four perceptive cycles are presented in Fig 5

The perceptive cycles increase the recognition rates. After 4 cycles, the classifier reaches $91.7 \%$. A TNN without perceptive cycles is worse than a MLP (45.2\% instead of $81.6 \%$ ) because TNN does not have many constraints in its intermediate layers. With perceptive cycles, the context returns make it possible to gain in precision while the algorithm complexity increases with a 2.5 factor.

\section{Conclusion}

We presented in this article a neural network architecture for document logical structure analysis. The method uses a Transparent Neural Network that makes it possible to introduce knowledge in each neuron and to organize in hierarchy the neurons in order to create a "vision" decomposition. The topology can simulate a decomposition hierarchy from fine (the patterns to recognized) to coarse (the global context). 
Thanks to this system, we can adapt the computation time according to the pattern granularity and complexity. These "perceptive cycles" as named in cognitive psychology allow simulating in the same system a recognition process that uses automatic and fixed knowledge rules, a hierarchical view, and an interpretation-correction process thanks to hypothesis creation. An input feature clustering was done to speed-up the perceptive cycles.

The TNN gives encouraging results. Although some improvements are in hand, tests are already better than a simple MLP, without adding too heavy computation. In our future works, we will propose a genetic-method to choose representative samples in the database during the context return. Another work will be done to improve the feature subset creation and a method to deal with the final cases of rejected patterns will be presented.

\section{References}

1. Mao, S., Rosenfelda, A., Kanungo, T.: Document structure analysis algorithms: A literature survey. SPIE Electronic Imaging (2003)

2. Nagy, G.: Twenty years of document image analysis in pami. PAMI (2000)

3. Guyon, I., Elisseeff, A.: An introduction to variable and feature extraction. Journal of Machine Learning Research (2003)

4. Rangoni, Y., Belaïd, A.: Data categorization for a context return applied to logical document structure recognition. ICDAR (2005)

5. Cattell, R.: The scree test for the number of factors. Multivariate Behavioral Research (1966)

6. Zwick, W.R., Velicer, W.F.: Comparison of five rules for determining the number of components to retain. Psychological Bulletin (1986)

7. LeCun, Y.: (http://yann.lecun.com/exdb/mnist/)

8. Siggraph: http://www.siggraph.org/s2003/. (2003) 